Chapter 27

\title{
ICT SUPPORT FOR EVOLVING HARMONIZATION OF INTERNATIONAL ALLIANCES
}

Ronald M. Lee, Elizabeth Dominguez Campillo

Abstract: International alliances are agreements between multiple countries to cooperate on trade, or other forms of economic activity, for mutual benefit. This paper focuses on how information and communication technologies (ICT) can support transaction efficiency and effective controls in the context of evolving international alliances. We concentrate on the potential for electronic procedures to manage the document flows automatically, in a fashion analogous to workflow systems within organizations. The key challenge, however, is how to support the evolution of these documentary procedures to match the dynamic structure of international alliances.

\section{INTRODUCTION}

There is a saying that no man is an island - we are social animals and need to participate in social networks. The same, it seems, also applies to nations. More and more, nations are interlinking via various forms of international alliances. By international alliance we mean agreements between multiple countries to cooperate on trade, or other forms of economic activity, for mutual benefit. Examples include the World Trade Organization (WTO) which is the administrative organization that implements the General Agreement of Tariffs and Trade (GATT), European Union (EU), North American Free Trade Alliance (NAFTA), MERCOSUR (Argentina, Brazil, Paraguay, Uruguay), CARICOM (Caribbean countries), and many others. (Here we are not specifically concerned about military alliances, though they may share some aspects.) In some cases, such as the European Union, there may even take on the character of a government, with a president, and parliament. The purposes of these international alliances are varied, but typically include efforts to reduce transaction costs among their members and to achieve economies of scale in their markets. 


\subsection{HETEROGENOUS MEMBERSHIP; NEED FOR HARMONIZATION}

International alliances are inspired by good intentions among the initial members. Often they are motivated in response to some outside pressure or threat. But the implementation often becomes bogged down as the members realize the extent of their various differences on detailed, but yet relevant points. The goal, ideally, is a harmonious solution that allows the parties to inter-operate, yet enables each to maintain their distinctive mode of operation. This is of course not always possible, and compromises need to be hammered out. This can take time, especially if some of the parties are reluctant.

\subsection{EVOLUTIONARY}

Working out these various harmonious solutions and compromises can be slow and arduous - often taking years to complete. In the meantime, conditions may change requiring further revisions. These changes in policy can be:

a. monotonic -- adding new features without affecting existing features of the policy

b. obsolescent -- adding new features that make other features ineffective or obsolete

c. non-monotonic -- adding new features to the policy that actually contradict previous policies.

(these are the kinds most difficult to handle)

\subsection{POTENTIAL FOR TECHNOLOGY SUPPORT:}

The heterogeneity of international alliances is mainly due to the differences in social and economic values of the member countries. These are determined by various types of domestic political processes. However, the participation in international alliances can create issues that need to be addressed by these domestic processes. While the political process itself is outside the scope of this research, we want to address how information and communication technology (ICT) can facilitate the deployment and evolution of international alliances, especially in their more procedural aspects. Here we introduce the term "protocol" to encompass procedures, processes, documents and associated rules agreed upon by the international alliance. 


\subsubsection{NORMAL OPERATIONS}

The normal operation of documentary procedures for an international alliance includes the following features:

* computerized implementation of international alliance protocols

* facilitate learning and adoption of international alliance protocols

* deployment -- making international alliance protocols readily available and useable to all appropriate international parties

* secure -- controlling access, authentication as appropriate so that the international alliance protocols are not abused or used fraudulently

* monitoring usage and performance of international alliance protocols

\subsubsection{CHANGE, EVOLUTION MANAGEMENT}

The dynamic nature of international alliances also imposes the following additional requirements:

* detecting need for change (based on monitoring)

* helping to organize change proposals

* help to analyze, determine potential consequences and costs of changes

\section{EXAMPLE INTERNATIONAL ALLIANCE: CARICOM}

The Caribbean Community and Common Market (CARICOM) provides a typical example of an international alliance. It is a regional alliance to provide economic integration, hence reduction of transaction costs and economies of scale, for countries in the Caribbean region. It was established in 1973 under the Treaty of Chaguaramas. CARICOM replaced the former Caribbean Free Trade Association (CARIFTA). As is seen in various other international alliances, CARICOM's primary mandate is to provide a framework for regional, political and economic integration. Also common in other international alliances is the heterogenous status of membership, which presently has four levels: full members of both the community and common market; members of the community only; associate members; observers. Members of the Caribbean Community and Common Market include: Antigua \& Barbuda, Barbados, Belize, Dominica, Grenada, Guyana, Haiti, Jamaica, Montserrat, St. Kitts \& Nevis, St. Lucia, St. Vincent and the Grenadines, Suriname, Trinidad and Tobago. The Bahamas is a member of the Community, but not the Common Market. Associate members are British Virgin Islands and the Turks and Caicos. Observer nations include Anguilla, The Dominican Republic, The Netherlands Antilles, Puerto Rico and Venezuela. The objectives of CARICOM include the free movement of goods, services, persons, and capital throughout the region. These policies, 
and the current status of specific items is clearly stated in their Web site (www.caricom.org).

\section{CHALLENGE: FLEXIBLE, ADAPTIVE BUREAUCRACIES}

In the theory of organizations (e.g. Weber 1956/1978), the term 'bureaucracy' refers to management based on uniform, explicit rules, rather than case-by-case discretionary decisions. In this technical sense, international alliances are bureaucracies. Note: bureaucracies no longer need to be of the brick-and-mortar variety - they may also exist virtually. For instance, the North American Free Trade Alliance, NAFTA, is a virtual organization. A common complaint about bureaucracies is that they are inefficient. Requests are routed through numerous offices, each of which may have a backlog, leading to an accumulation of delays. Non-routine requests are sometimes misrouted, creating additional delays. Errors in the request cause backtracking and still further delays. Other complaints are about the effectiveness of the system. In order to keep the complexity manageable, bureaucratic rules may be simplistic, covering only standard cases. Exceptional requests are either rejected or diverted to a discretionary authority. When there are many exceptions, a change in regulations may be called for. However, because of the complexity already present, this is often difficult to do. Change is either resisted or made minimally.

On a broader scale, the very existence of a bureaucracy is sometimes criticized. Bureaucracies are often erected to perform a particular governmental or social function, and are thus protected from competitive challenges. They may grow to such size that the sheer momentum of their economic weight relative to the society carries them forward. An important aspect of this problem is a failure to effectively manage complexity. Bureaucracies are "sticky upwards". That is, they seem to grow more easily than they shrink. Nearly three decades ago, Elgin and Bushnell (1977, p. 337) complained that "we have rushed to create bureaucracies of such extreme levels of scale, complexity, and interdependence that they now begin to exceed our capacity to comprehend and manage them." Today, despite all the remarkable advances that we have seen in information and communication technologies, these complaints seem just as appropriate. Furthermore, this is certainly not because bureaucracies have ignored these advances. Indeed, bureaucracies are among the most aggressive consumers of ICT. Certainly, ICT has done much to improve bureaucratic efficiency. On the other hand, we claim, it has not helped at all in the management of bureaucratic complexity. Indeed, it has aggravated the problem by obscuring bureaucratic rules and procedures in the form of computer codes. 
(For instance, many of us have experienced the frustrations of trying to rectify a billing problem that is computerized.)

This is both a representation problem and a modeling problem. It is a representation problem in that computerized rules and procedures need to be made more accessible and directly controllable by management. It is a modeling problem in that improved methods of systems analysis are needed to more effectively analyze and design complex bureaucratic systems. This is not, we argue, merely a matter of refining present techniques. There is a need for more fundamental revisions in our approach to such problems (Stamper, 1979; Lee, 1985; Kimbrough and Lee, 1986). Bureaucratic systems are not merely information processing systems. They are systems of organizational and social control. They convey more than data; they convey orders, commands, obligations, contracts, permissions, licenses, vouchers, receipts, prohibitions, waivers, verifications, etc. etc. etc. These are performative transactions in that by their communication they change the nature of social relationships within the organization.

These problems are well-recognized in large rationalized organizations and governmental agencies. However, we argue that these kinds of problems are most salient for international alliances, which are an evolving dialectical balance among multiple national interests.

\section{APPLICATIONS SOFTWARE AND ORGANIZATIONAL CHANGE}

It is a commonplace observation that organizations, to survive, must adapt to changes in their environment. Those that do not are forced out of business, if they are companies in a competitive market; have their budgets canceled, in the case of government bureaucracies; or are overthrown, in the case of governments themselves. Just how an organization should be designed to accommodate change is, of course, a much more difficult matter, and has been the subject of many volumes of organizational theory. One aspect of this general problem seems to have been neglected, namely, the effect of information technology on the organization's ability to adapt and change.

Certainly, there are numerous clear cases where the installation of an information system adds to the organization's flexibility. For instance, the installation of a centralized database may allow data to be accessed and combined in a variety of ways that would have been practically impossible when that data was recorded in paper files scattered throughout the company. The flexibility of a given computer application obviously depends on the foresight of its designers. To this end, programming students are generally taught to seek the most general definition of the problems they are 
given so that the resulting program can handle not only the immediate problem but also variants of it that might arise.

This strategy has obvious limitations. In seeking to find a generalized solution, the programmer may waste undue amounts of time on conditions that will never arise. He/she must therefore make a choice as to how much flexibility to encode into the program logic. We refer to the level of flexibility chosen as the 'designed flexibility' of the system. Selecting the appropriate level of designed flexibility is however difficult and, almost certainly, new requirements will later arise that were not planned for originally, so that the program must be modified. This is where the problem arises. Anyone who has written even small programs will know that it is much easier to incorporate a given feature in the program logic in its original writing rather than try to add this feature afterwards. This difficulty rises exponentially with the complexity of the original program or system.

To summarize, the basic problem with current application systems is that they are 'brittle'; i.e. they cannot easily be reformed to adapt to changing circumstances. This brittleness has profoundly disturbing consequences as more and more organizations, ranging from small and medium size companies to immense governmental agencies, convert their information processing to computer software. The immediate gains of increased efficiency, speed of processing, rapid access to centralized data files, etc., are clear (or the investment would not be justified). However, there may be a long term, possibly devastating hidden cost as the organization finds its ability to adapt and respond to new environmental conditions hampered by its inability to modify its information systems accordingly.

Concerns such as these have been major motivations in the development of object oriented programming languages (including Java) and, componentbased architectures.

\section{SOFTCODED ELECTRONIC PROCEDURES}

As illustrated by the example of CARICOM, international alliances typically deal with the free movement of goods, services, persons, and capital among the countries that are party to the alliance. One may view this as a transaction simplification process, reducing the bureaucratic overhead of these kinds of exchanges. However, this process of simplification tends to be a gradual one, over an extended period of time.

To give a sense of the scope of complexity that is involved, consider the procedure in Figure 1, which is for the import/export of goods through a seaport. This is a typical procedure, not involving added complexities of dangerous goods, animals, foodstuffs, or terrorist controls.

A point that we want to emphasize here is that the reduction of bureaucratic requirements can be complemented with the introduction of 
ICT solutions, which can make the existing bureaucratic overhead much less costly and time consuming.

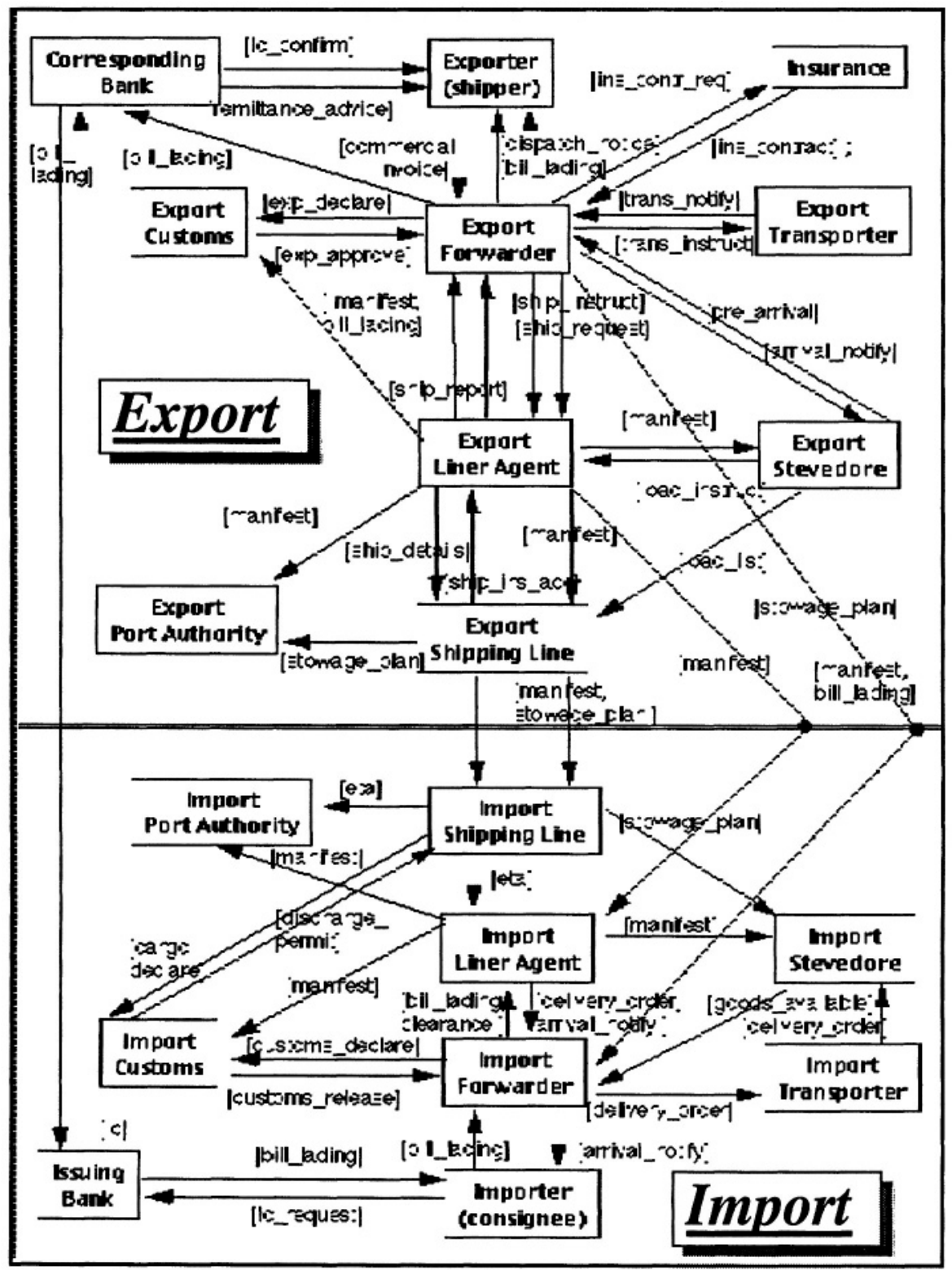

Figure 1. Typical International Trade Transaction

We might illustrate this point with a comparison to a standardization of keyboard layout for Latin alphabet languages. The US and UK have a 
keyboard standard called QWERTY, which refers to the keys in the left side of the top alphabetical row. The French prefer a layout that is AZERTY. During the time of Salazar, Portuguese adopted a standard layout HCESAR. At one time, the choice of a standard keyboard layout was a point of serious debate. Eventually, however, computers became flexible enough to support any variety of keyboard layouts, simply by changing software. Thus, the advance of technology made the problem disappear.

In a similar way, we believe that software solutions can also help to eliminate negotiation obstacles for international alliances. That is, instead of everyone agreeing to a single standardized solution, there may be a harmonized approach that allows existing national standards to inter-operate, with a minimum of adaptation. In many cases, software has already been developed to automate international procedures at a national level. However, it is likely that the procedures have been 'hard coded' into the application software - that is, the sequence of procedural steps is expressed in the program code. A key aspect of the architecture presented here is that the international procedures are 'soft coded', in a declarative, rule-based form. Application software then needs to be made generic, not committed to a particular version of the procedure. Instead, the application will interpret the procedure from an external specification (analogous to the idea of 'tabledriven' software).

Within a national context, this has an immediate benefit of expediting the deployment of changes to procedures. Rather than sending out new versions of manual instructions (for non-automated procedures) or new versions of software (hard-coded programs), one may simply distribute a revised representation of the procedure, or have it accessed from a central repository - as illustrated in Figure 2. Even more significant is that such procedures can be analyzed and managed using computational tools. For example, analytical techniques can be applied to check for formal correctness (boundedness, etc.), as well as fraud potential and other audit controls. Further, soft-coding allows for the representation of generic models that are parameterized for specific circumstances. Additionally, soft-coding enables the navigation, synthesis and negotiation of procedures from different trading sectors or regulatory environments.

\section{REPRESENTATION OF DOCUMENTARY PROCEDURES}

We found Petri Nets to be the best formal representation for procedures that offer both a graphical representation and a formal basis for the verification of various properties of these procedures. The main advantage of the Petri Net formalism, in addition to its capability to graphically model both concurrency and choice, is that it offers various kinds of both formal 
and informal analysis methods, which make Petri Nets especially suitable for modeling "Discrete Dynamic Systems" (van der Aalst, 1992). We have extended the Petri Net representation to include the exchange of documents (as document places) in what we call Documentary Petri Nets (DPN's). This DPN representation is explained in detail in Lee and Bons (1996). Figure 3 presents an example Documentary Petri Net, as modeled using the graphic design environment InterProcs (see www.euridis.nl/?view=Interprocs). This system produces executable electronic procedures that are decomposed by role to execute autonomously in each of the separate organizations involved in the transaction.

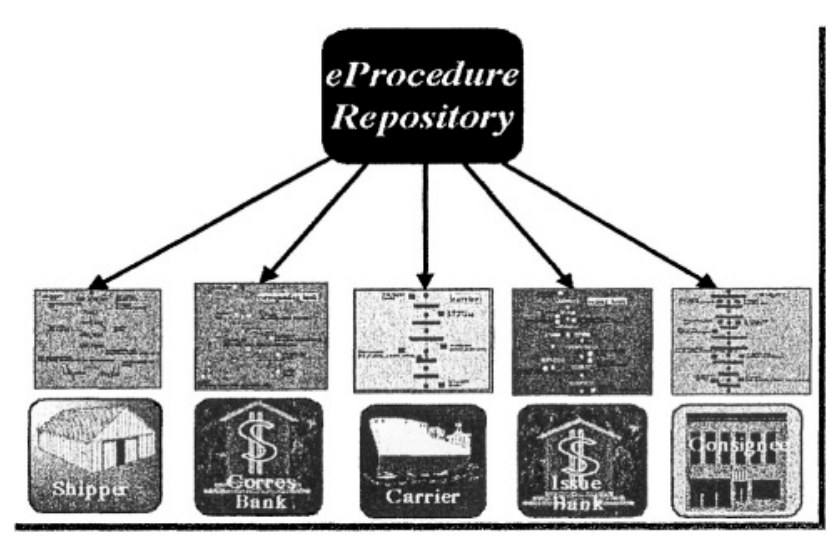

Figure 2. Parties Downloading from eProcedure Repository

These observations highlight the focus on aspects of multi-lateral harmonization as a priority for future research. A technical solution to enable multi-lateral harmonization that we describe is the agent-based approach called the Messenger Model. Further refinement of this technology focuses on the constraint resolution mechanism for Procedure Constraint Grammars (PCG's) (Lee, 2001).

Another research direction relates to normative modeling of documentary procedures: given the goals and control concerns of the various member countries for a particular type of transaction, what constitutes a 'good' procedure, which balances the excess transaction costs of over-control against the risks of under-control? This direction of future research will pursue development of automated verification tools that may be used to check whether a proposed documentary procedure conforms to specified control requirements. An advantage of using Petri nets as the basis of the DPN formalism is that various kinds of automated verification algorithms and tools already exist, for instance to detect possible dead-lock situations. However, our modeling experience has shown that these are of limited usefulness in practical situations - these kinds of problems seldom arise, and 
if they do, are usually quite obvious. The more serious control problems have to do with fraud and collusion, which require a deeper semantic understanding of what the procedure is actually doing. An approach to this is the notion of "audit patterns", originally developed by Lee (1991), and elaborated in the dissertation work of Chen (1992) and Bons (1997). Further research opportunities exist in this direction.

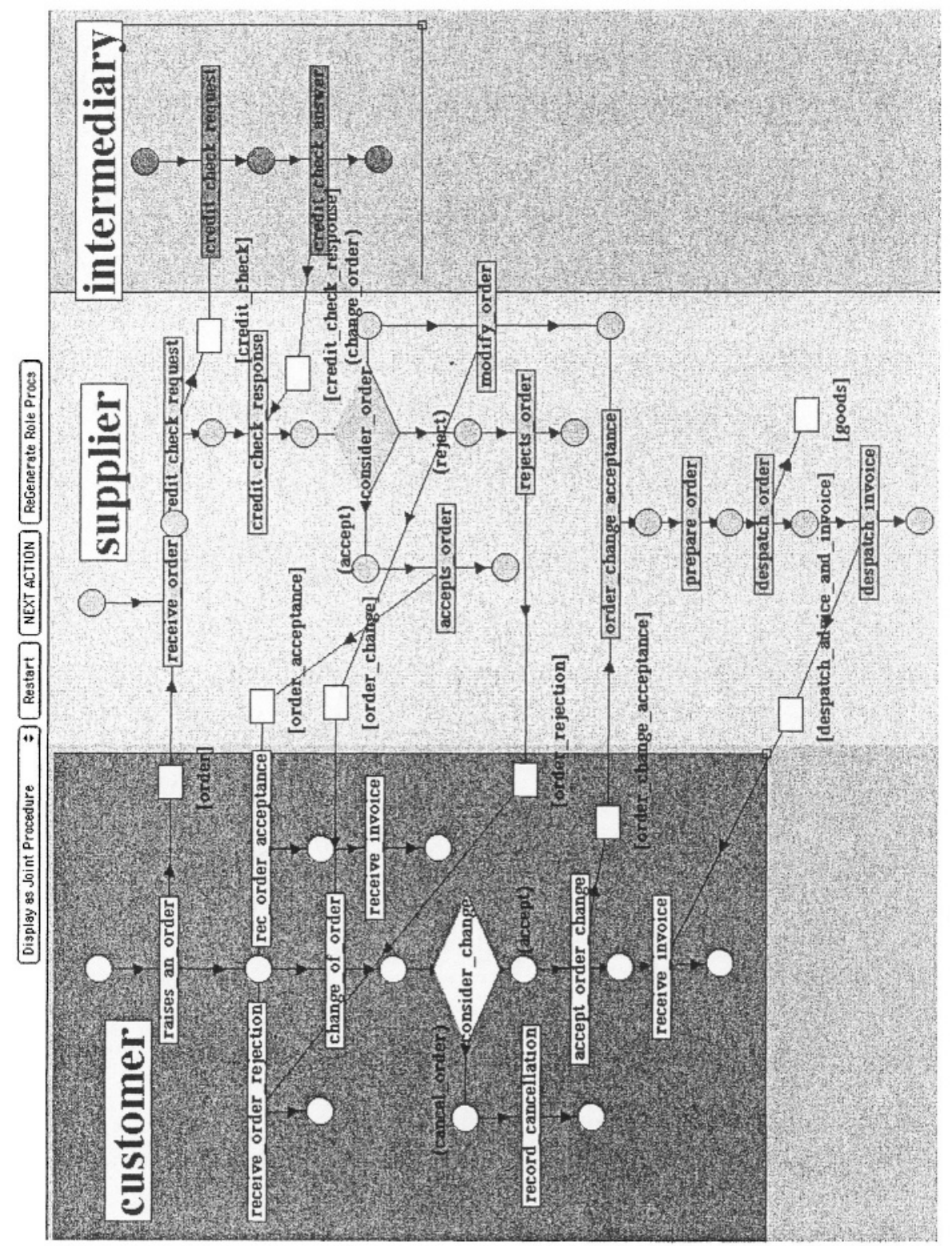

Figure 3. InterProcs Display of Order Process as DPN 


\section{REFERENCES}

Aalst, W.M.P. van der "Timed coloured Petri Nets and their application to logistics", PhD thesis Eindhoven University of Technology, 1992

Austin, J.L."How to DO things with words", Harvard University Press, Cambridge, MA, 1962

Bons, Roger W.H., Designing Trustworthy Trade Procedures for Open Electronic Commerce, $\mathrm{PhD}$ Dissertation, Euridis and Faculty of Business, Erasmus University, September, 1997.CARICOM, see www.caricom.org.

Chen, Kuo-Tay. Schematic Evaluation of Internal Accounting Control Systems, University of Texas at Austin, PhD Dissertation, 1992.

Chen, KuoTay and Lee, Ronald. "Schematic Evaluation of Internal Accounting Control Systems", Euridis Research Monograph (RM 92.10.01), October, 1992.

Dewitz, S. K. and Lee, R. M. "Legal Procedures as Formal Conversations: Contracting on a Performative Network", Proceedings of International Conference on Information Systems, Boston, December 1989, pp. 53 - 65.

Elgin, D.S. and Bushell, R.A. 1977. "The Limits to Complexity: Are Bureaucracies Becoming Unmanageable?", The Futurist, December.

Genrich, H.J. and Lautenbach, K. "System Modelling with High-level Petri Nets", Theoretical Computer Science, Vol. 13 pp 109-136, New York: North-Holland, 1981

Genrich, H.J. and Lautenbach, K. "The Analysis of Distributed Systems by Means of Predicate/Transition Nets", Semantics of Concurrent Computation: Lecture Notes in Computer Science, Kahn G. (Ed.), Vol. 70 pp 123-146, Springer Verlag, 1979

ICC, "The Uniform Customs and Practices for Documentary Credit Procedures", International Chamber of Commerce publication 500, Paris, France, January 1994

Kimbrough, S.O. and Lee, R.M. "On Illocutionary Logic as a Telecommunications Language", Proceedings of the International Conference on Information Systems (San Diego; December, 1986)

Lee, R.M. 1985. "Bureaucracy as Artificial Intelligence", in Humphreys, P.(ed.) Knowledge Representation for Decision Support, Proceedings of IFIP WG 8.3 Working Conference (Durham, England; July, 1984), North-Holland.

Lee, R.M. "Auditing as Pattern Recognition: Automated Analysis of Documentary Procedures", Working Paper, Department of Management Sciences and Information Systems, University of Texas at Austin, August 1991.

Lee, R.M. "Automated Generation of Electronic Procedures: Procedure Constraint Grammars" Decision Support Systems, Special Issue on Formal Models for Electronic Commerce, eds Kimbrough, S. and Tan, Y-H, 2001, pp. 291-308.

Lee, R.M. and Bons, R.W.H, "Soft-Coded Trade Procedures for Open-EDI" International Journal of Electronic Commerce, Vol 1, No 1, 1996, pp. 27-49.

Lee, R.M., Bons, R.W.H, Wagenaar, R.W. "Pattern-Directed Auditing of Inter-organisational Trade Procedures" Proceedings of the 1st IFIP Conference on eCommerce, eBusiness, and eGovernment, Zurich, Switzerland, 4-5 October 2001.

Peterson, J. L. "Petri Net Theory and the Modeling of Systems", Prentice-Hall, 1981 
Petri, C.A. "Kommunikation mit Automaten", PhD thesis University of Bonn, Germany, 1962.

Searle, J. "Speech Acts: An Essay in the Philosophy of Language", Cambridge University Press, London, 1969

Stamper, R. 1979. "LEGOL as a Tool for the Study of Bureaucracy", Proceedings of the IFIP TC8 WG 8.2 Conference on the Interaction of Information Systems and the Organization.

Weber, M. 1956/1978. Economy and Society. Berkeley, California: University of California Press, translated from Wirtschaft und Gelsellschaft. Tuegingen: J.C.B. Mohr, 1956. 